Editorial

\title{
Good documentation practices (GDPs) in pharmaceutical industry
}

Volume 4 Issue 2 - 2017

Krishan Kumar

Department of Radiology, The Ohio State University, USA

Keywords: good documentation practice, pharmaceutical products, compliance, quality assurance, SOP, GLP, cGMP, GCP, FDA, FMA, WHO

Abbreviations: GDP, good documentation practice; USP, united states of pharmacopeia; WHO, world health organization; GLP, good laboratory practice; GCP, good clinical practice; SOPs, standard operating procedures

\section{Editorial}

A written, a printed, a magnetic, or an electronic media containing information or data related to a formulation and a manufacturing process for a product constitutes a document or record. Documents must be clearly and legibly written or created, must be traceable, and provide enough details of the activity and accurate history of the event. Documents or records provide evidence that the products are manufactured according to the pre-developed processes and the predefined specifications.

Good Documentation Practice (GDP or GDocP), a term used in the pharmaceutical industry, is essential for the integrity of data collection and reporting for supporting development, registrations, commercialization, and life-cycle management of pharmaceutical products. ${ }^{1}$ Adhering to the GDPs assures preventing errors within the manufacturing environment and during the analysis of pharmaceutical products which could otherwise impact product quality, and safety of the patients, state of manufacturing facilities, and related activities. Compliance to GDPs is required by both the US and the European regulatory authorities, i.e., FDAs CFR (Code of Federal Regulations) and EMA (European Medicines Agency). In addition to the United States of Pharmacopeia (USP) issuing a general chapter $<1029>,{ }^{2}$ the World Health Organization (WHO), ${ }^{3}$ Health Canada, ${ }^{4}$ and EudraLex (the collection of rules and regulations governing medicinal products in European Union) $)^{5}$ have published specific guidance related to GDPs. On the other hand GDP is an important part of current Good Manufacturing Practices (cGMPs) in the US. ${ }^{6}$ In addition to regulatory requirements, it is also important to maintain accurate records and documentations of activities in the pharmaceutical industry for business reasons which allow one to critically evaluate the internal procedures for improvement of processes and products and spending less time in repeating studies, if needed, rather than starting from the beginning.

The regulatory authorities inspect pharmaceutical development and manufacturing facilities for compliance of good laboratory practice (GLP), good clinical practice (GCP), and cGMP. During these inspections, the inspectors audit the pharmaceutical companies for bad documentation practices including incomplete records, disorganized documents, lack of compliance to the Standard Operating Procedures (SOPs), inconsistent documents, non-validated electronic systems, non-certified copies, poor correction practices etc. Inadequate and poor documentation practices may result citations in the form of FDA's 483 observation(s) and/or warning letters. Consequences of both may be time consuming and costly including time spent in responding to the observations and potentially making some operational changes. Moreover, this information is in public domain and may be detrimental to the reputation of the organization. In the recent years, majority of the $483 \mathrm{~s}$ and warning letters issued by the FDA had observations related to data integrity and documentation practices. These deficiencies may have resulted from the organizational timeline pressures, lack of expertise, resources, and attention and commitment to the tasks, redundant and out-of-date processes and controls, and limited guidance and training.

As per the $\mathrm{WHO},{ }^{7}$ the purpose of Good Documentation Practices is

a. To define the specifications and procedures for all materials and methods of manufacture and control,

b. To ensure that all personnel know what to do and when to do it,

c. To ensure that authorized persons have all information necessary for release of a product,

d. To ensure the existence of documented evidence, traceability, and to provide records and an audit trail for investigation and

e. To ensure the availability of data for validation, review, and statistical analysis.

Some examples of documentations used in the pharmaceutical environment are (but not limited to): Laboratory Note Books, Batch Record, Bills of Materials, Specifications, Policies, Protocols, Standard Operating Procedures (SOPs), Work Instructions, Test Methods, Check Lists, Forms/Log Sheets, Training Assessments, Electronic and Hardcopies of Quality Records, Certificate of Analysis, Certificate of Compliance, Technical Transfer Reports, Validation Documents etc. etc. 
Documents should be designed, prepared, reviewed, and distributed for use per established procedures. These should have characteristics of being attributable, legible, contemporaneously recorded, original, and accurate (referred as ALCOA). There are numerous publications available that describe the procedures of documentation including what to do and what not to do. Some of the examples in the to do list are: using black or blue indelible ballpoint ink, making entries legible, initialing and dating all entries, documenting each step before moving to the next step, crossing out unused space, signing the document at the end of the event, etc. Similarly, some examples of not to do are: overwriting, using liquid correction, backdating, recording data before the event occurred, using scratch paper from recording, leaving blank spaces etc.

Documentation should be as detail as possible. A list of some examples (but not limited to) to be included in the document are: purpose of the study (including study type, e.g., good laboratory practice (GLP), good clinical practice (GCP), or good manufacturing practice (GMP), discovery, developmental, method development/ validation, formulation/process development, pharmaceutical analysis, bioanalysis, metabolism, and stability, etc.), protocol or study number, materials (source and lot/batch number), standards, and the results/conclusions etc. A complete record and documentation will demonstrate accuracy and completeness of the data collected and reported ensuring the integrity of the data and ability to reproduce the study, if needed.

It is important and essential to establish procedures for archiving and safeguarding of documents so that these are available during repeat of studies, if needed, during product registrations, and during inspections by the regulatory agencies. The organizations should establish appropriate infrastructure, written policies and procedures, and systems to prevent any loss or damage to the documentations during archival, retrieval, and transfer from one department to another.

In summary, as a good business practice and to be compliant with the expectations of the regulatory authorities, it is important that an organization

a. Provides enough resources to create and complete documentation,

b. Ensures that the documents are attributable, legible, contemporaneously recorded, original, and accurate, c. Conducts a thorough gap analysis of existing documentation for any missing documentation or revision,

d. Provides resources for initial and ongoing training to the personnel, involved in development and manufacturing of the pharmaceutical products, in documents creation, management, and security of documents during lifecycle management of products. Finally, an oversight of Quality Assurance is needed to ensure that the personnel are following the organization SOPs.

\section{Acknowledgments}

None.

\section{Conflicts of interest}

Author declares there are no conflicts of interest.

\section{Funding}

None.

\section{References}

1. Kumar K. Data Integrity in Pharmaceutical Industry. J Anal Pharm Res. 2016;2(6):00040.

2. USP. Good Documentation Guidelines, US Pharmacopeial Convention, USA. 2015.

3. WHO. Guidance on Good Data and Record Management Practices, World Health Organization, Switzerland. 2016. p.165-210.

4. Health Canada. Good Manufacturing Practices Guidance Document. Health, Canada. 2015.

5. EodreaLex-European Commission. Good Manufacturing Practices for Medicinal Products for Human and Veterinary Use. European Commission Health and Consumers Directorate-General. Belgium. 2011;4:1-9.

6. (a) FDA. The Code of Federal Regulations Title 21, Part 210-Current Good Manufacturing Practice in Manufacturing, Processing, or Holding of Drugs. US Food and Drug Administration, USA. (b) US FDA (201) The code of Federal Regulations Title 21, Part 212-Current Good manufacturing Practice for Finished Pharmaceuticals. US food and Drug Administration, USA. 2016.

7. WHO. World Health Organization Module 12, Part 1. 2006. 